\title{
Analysis of Clinical Characteristics of Submucosal Tumor of Digestive Tract Under Endoscope
}

\author{
Weixiang Ye ${ }^{1}$, Zhaolin Chen ${ }^{2, *}$ \\ ${ }^{1}$ Endoscopy Center, The First Affiliated Hospital of Jinan University, Guangzhou, China \\ ${ }^{2}$ Gastroenterology Department, The First Affiliated Hospital of Jinan University, Guangzhou, China
}

Email address:

ywx2200@126.com (Weixiang Ye),2009gzjnu@163.com (Zhaolin Chen)

${ }^{*}$ Corresponding author

\section{To cite this article:}

Weixiang Ye, Zhaolin Chen. Analysis of Clinical Characteristics of Submucosal Tumor of Digestive Tract Under Endoscope. International Journal of Biomedical Science and Engineering. Vol. 9, No. 1, 2021, pp. 1-5. doi: 10.11648/j.ijbse.20210901.11

Received: December 30, 2020; Accepted: January 11, 2021; Published: January 22, 2021

\begin{abstract}
Background: Submucosal tumors of the digestive tract are common diseases, which are difficult to diagnose and treat because of their occurrence in the digestive tract. In the past, follow-up observation or surgical operation were usually adopted for SMT, but the surgical operation had many disadvantages such as large trauma, many complications and long postoperative recovery time. In contrast, endoscopic therapy is characterized by safety. The clinical features of endoscopic mass resection remain unclear. Objective: To analyze the clinical features of submucosal masses of digestive tract. The pathological types and distribution of the tumor and the safety of endoscopic surgery were also discussedMethods: We analyzed the clinical data of 108 patients with submucosal tumors of the digestive tract by endoscopic surgery in our hospital. Results: The submucosal masses of the digestive tract were mainly leiomyoma and gastrointestinal stromal tumor, mostly benign lesions, mainly distributed in the mucosa and muscularis propria, and there was no difference between the sexes. The operative complication rate of endoscopic surgery for submucosal masses of digestive tract is low. Conclusion: $\mathrm{c}$ of small gastric submucosal tumors is safe enough and effective in diagnosis and treatment. EMR and ERE are still commonly used endoscopic surgery methods at present.
\end{abstract}

Keywords: Submucosal Tumor, Endoscopic Resection, EMR

\section{Introduction}

Submucosal tumor (SMT) of the digestive tract refers to the pathological changes below the gastrointestinal mucosa, most of which are benign, but some of which have the potential for malignant transformation [1]. Common SMT includes mesenchymal fibroids, plasmas, phlebiomas, neuroendocrine tumors, lipomas, and ectopic pancreas [2-5]. However, there were differences in the nature of masses in different parts of the digestive tract, and leiomyoma and stromal tumor were more common SMT lesions in the esophagus, stomach and duodenum, etc, but the distribution of various digestive tract masses in patients of different ages was still less reported.

In the past, follow-up observation or surgical operation were usually adopted for SMT, but the surgical operation had many disadvantages such as large trauma, many complications and long postoperative recovery time [6]. In contrast, endoscopic therapy is characterized by safety, minimally invasive, good curative effect, less pain and low cost, and has absolute advantages compared with traditional surgical treatment. In recent years, with the development of endoscopic technology, some surgical diseases have become indications for endoscopic treatment of digestive tract, especially the promotion of titanium clips, which can be used to perform full-thickness resection of deeper tissues under the endoscope [7]. Endoscopic submucosal dissection (ESD), endoscopic mucosal resection (EMR), submucosaltunnel endoscopic resection (STER) and endoscopic submucosal excavation (ESE) technique for successful, on the basis of endoscopic after submucous resection of the neoplasm can complete resection for gastrointestinal SMT can effectively prevent leakage of abdominal digestive juices at the same time [7].

After endoscopic technology made breakthroughs in the early diagnosis of digestive tract, HOSOKAWA and others 
were the first to use ESD technology for endoscopic treatment of early gastric cancer [8]. At present our country endoscopic cancer early diagnosis rate, incidence of postoperative complications, and treatment situation reports there are still large differences, therefore, this research will be to our hospital in recent 2 years at a single endoscopy center line endoscopic gastrointestinal SMT cases were retrospectively analyzed, analysis the clinical features of endoscopic gastrointestinal submucosal lesions and report accordingly.

\section{Materials and Methods}

\subsection{General Data}

108 patients with upper gastrointestinal SMT who were hospitalized and treated with EMR, ESD, ESE or STER in our hospital from March 2016 to December 2020 were retrospectively collected. There were 53 males and 55 females. Age $24 \sim 85$ years old; The lesions were located in the esophagus in 36 cases: 10 cases in the upper segment, 20 cases in the middle segment, 6 cases in the lower segment, 72 cases in the stomach: 2 cases in the cardia, 28 cases in the gastric fundus, 23 cases in the gastric body, 17 cases in the gastric antrum, and 2 cases in the gastric Angle. All patients were suspected to have submucosal tumors by endoscopic examination, and the nature of lesions was preliminarily determined and the level and scope of lesions were confirmed after re-examination by endoscopic ultrasonography and CT. Therefore, the patient excluded the invasion and metastasis of the digestive tract, and excluded the contraindications through the examination of coagulation function, cardiopulmonary function and so on. All patients were informed of the potential benefits and associated risks of endoscopic surgery before surgery, and were required to sign an informed consent.

\subsection{Methods}

\subsubsection{Surgical Methods}

All patients signed the informed consent of surgical risk, fasting for $8 \mathrm{~h}$ before surgery, water deprivation for $4 \mathrm{~h}$ before surgery, intravenous antibiotics for $30.0 \mathrm{~min}$ before surgery to prevent infection. All operations were performed under tracheal intubation and general anesthesia, and $\mathrm{CO}_{2}$ air pump was used in the operation. The most suitable surgical method was adopted for different tumors. The general steps of the operation are as follows: To locate and label the lesions: to attach a transparent cap to the head of the gastroscope before the treatment, to search for tumors under the endoscope, and to accurately locate the lesions; (2) Fully raise the lesions: multi-point submucosal injection of liquid was performed around the lesions to completely raise the lesions, and $0.4 \%$ indigo carina $2 \mathrm{~mL}$ and $0.9 \%$ sodium chloride solution $250 \mathrm{~mL}$ were mixed. Peripheral submucosal injection was performed outside the marking points to lift the lesions. (3) Cutting along the mucosal edge: Different medical instruments, such as Dual knife, Hook knife or IT knife, are used to cut the mucosa and submucosa along the above mark points; (4) Dissection and excision: The combination of various surgeries was used for the dissection between the submucosa and the outer edge of the tumor, to maintain the integrity of the capsule, the tumor was completely removed, pay attention to avoid damage to the serosal layer; (5) Postoperative wound treatment: treatment of bleeding foci and small visible blood vessels with large area or deep invasion of wounds with hot biopsy forceps with argonplasma plasmacoagulation (APC) should be performed to prevent the occurrence of postoperative perforation. Endoscopic hemostasis is feasible in the case of delayed hemorrhage.

\subsubsection{Treatment}

After surgery, patients were fasted for $24-48 \mathrm{~h}$ after surgery, and their vital signs were monitored to observe whether the patient had symptoms of chest tightness, shortness of breath, cyanosis, fever, abdominal pain and abdominal distension. If the patient had no obvious discomfort, the vital signs were stable, and the patient was gradually transferred to a liquid diet Postoperatively, proton pump inhibitor (PPI) was routinely administered with acid inhibition, gastrointestinal decompression and nutritional support. It is recommended that patients be followed up with endoscopy 1, 3 and 6 months postoperatively to observe whether the wound and tumor remain or not.

\subsection{Observation Indexes}

The observation indexes of this study included tissue stratification sources, pathological types, and surgical complications including bleeding, perforation, abdominal discomfort, age stratification, etc.

\subsection{Statistical Analysis}

SPSS20.0 software was used for statistical analysis of the data. Measurement data consistent with normal distribution were expressed as mean \pm standard deviation $(\mathrm{x} \pm \mathrm{s})$, and comparison between groups was performed by $\mathrm{T}$ test. Enumeration data were expressed as frequency or rate, and the $\mathrm{x}^{2}$ test was used for inter-group comparison. $\mathrm{P}<0.05$ was considered statistically significant.

\section{Results}

\subsection{Clinical Features of 108 Patients with Submucosal Masses of Digestive Tract}

In this study, there was no significant difference between the ages of male patients and female patients $(53.72 \pm 0.76 \mathrm{vs}$. $54.48 \pm 2.37, \mathrm{P}=0.747)$, and the ratio of male to female patients was close to 1:1 (Figure 1A). Distribution of esophageal mass and gastric mass: 10 cases in the upper esophagus, 20 cases in the middle esophagus, and 6 cases in the lower esophagus; There were 2 cases of gastric cardia, 28 cases of gastric fundus, 23 cases of gastric body, 17 cases of gastric antrum, and 2 cases of gastric Angle (Figure 1B-C). 




B
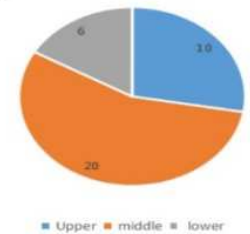

C

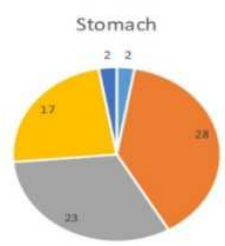

- Cardia = fundus = gastric boody = antrum = gastric Ange

Figure 1. Clinical features of 108 patients.

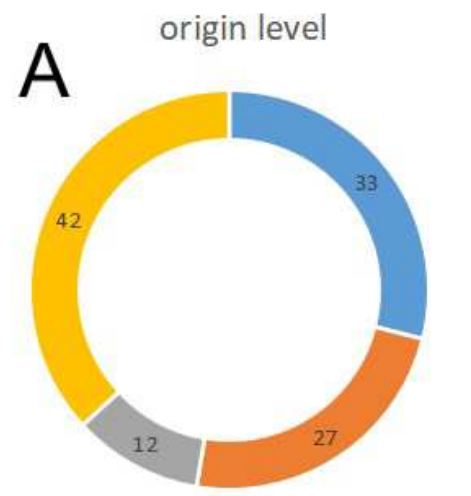

mucous layer

muscularis mucosae

- submucosa

muscularis propria

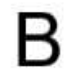

pathological pattern



$18 \%$
C

\section{Pathological pattern}

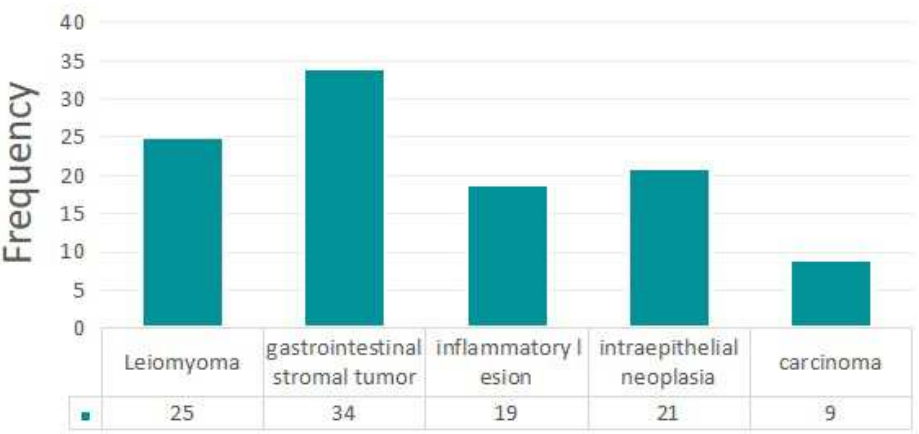

Figure 2. The distribution of pathological types of masses in different sites.

\subsection{Surgical Methods and Complications}

Table 1. Surgical methods of the 108 patients.

\begin{tabular}{ll}
\hline Surgical methods & Cases (\%) \\
\hline EMR & $44(40.7 \%)$ \\
ESD & $20(18.5 \%)$ \\
ESE & $39(36.1 \%)$ \\
STER & $5(4.6 \%)$ \\
\hline
\end{tabular}

ESD: endoscopic submucosal dissection, EMR: endoscopic mucosal resection, STER: submucosaltunnel endoscopic resection, ESE: endoscopic submucosal excavation.

Statistical analysis was performed on the surgical methods of all patients in this study, and the results showed that EMR and ESE were the main surgical methods for submucosal masses of the digestive tract (Table 1). Further analysis of the

\subsection{The Distribution of Pathological Types of Masses in Different Sites}

In order to analyze the distribution of gastrointestinal mucosal masses at different mucosal origin levels and their pathological types, pathological data of 108 patients were statistically analyzed, and it was found that 33 patients had mucosal masses, 27 patients had mucosal muscularis, 12 patients had submucosal masses, and 42 patients had proprioception (Figure 2A). Subsequently, the malignant degree of the tumor was analyzed, and it was found that malignant cases accounted for $18 \%$ in this study (Figure 2B). Further analysis revealed that the submucosal masses of the digestive tract were mostly leiomyoma and gastrointestinal stromal tumor (Figure 2C). 
unlikely to be completely and safely removed by standard endoscopy. Therefore, ER has not been a conventional indication in the past for most GSMT cases, especially for gastroenterointestinal stromal tumor (GIST) [9, 10].

However, it has recently been reported that endoscopic musculotomy/dissection and endoscopic full-thickness resection are minimally invasive treatments originating from the musculotomy propria with G-SMT [11, 12]. These from endoscopic submucosal dissection (ESD) technology of surgery for patients with G - SMT seems to be effective and safe, in reward in this study, we reported the endoscopic surgery including EMR, ESD, ESE or STER treatment cases of SMT, the above operation method in recent years has gradually been reported for the diagnosis and treatment of SMT, gradually shows the efficacy and safety of the operation [13]. In this report, the incidence of bleeding after treatment was about $25 \%$, and there was only one case of perforation. Moreover, all the bleeding cases were within the range of internal medicine control, and no secondary surgery or surgical intervention was required, which proved the safety and effectiveness of current endoscopic treatment of SMT. Studies have statistically found that the bleeding rate of endoscopic mucosal resection is $1.3 \% \sim 11.9 \%$, and most of the bleeding occurs during or within $24 \mathrm{~h}$ after surgery [14]. Bleeding is closely related to the site and size of the tumor.

Perforation is also a common complication, and the incidence of ESD related perforation is much higher than EMR, which is generally $1.2 \% \sim 9.7 \%$ [15]. The incidence of perforation in patients in this study is far lower than the reported incidence, which also proves that the safety of endoscopic techniques can be verified.

Most of SMT are benign tumors, but some of them have the potential of malignant transformation. Common SMT includes interleiomyoma, gastrointestinal stromal tumor, venous tumor, neuroendocrine tumor, lipoma, ectopic pancreas, etc $[2,3]$. But the nature of the different part of gastrointestinal neoplasm, reported finding more common SMT lesions of the esophagus, stomach and duodenum is leiomyoma, for gastrointestinal smooth muscle layer caused by abnormal growths, usually originated in mucosal muscularis and benign tumor, and to the muscularis propria in the reported 108 cases in our study, the main pathological type of leiomyoma and gastrointestinal stromal tumor, mainly distributed in the mucosa and, to the muscularis propria benign lesions in the majority, malignant lesions (18\%) are similar to most of the reports. Although gastric malignancy presenting as submucosal mass is rare, the incidence is approximately $0.1 \%$ to $0.6 \%$ [16]. Adenocarcinoma is covered with normal mucosa, and endoscopic mucosal biopsies usually do not reach the deep layer containing the cancer cells. The above also proves the feasibility and safety of endoscopic diagnosis of malignant gastrointestinal tumors.

\section{Conclusion}

In the cases we observed, the gastric submucosal masses were still more common as leiomyoma and gastrointestinal stromal tumorwe, and it was found that malignant cases accounted for $18 \%$ in this study. We believe that endoscopic resection of small gastric submucosal tumors is safe and effective in diagnosis and treatment. EMR and ERE are still the commonly used endoscopic surgery methods. Endoscopic surgery has a good ability to recognize malignant tumors in the diagnosis and treatment of G-SMT.

\section{Acknowledgements}

I am particularly grateful to my colleagues at the Endoscopy Center of the First Affiliated Hospital of Jinan University for their important assistance in the successful publication of this study. In addition, I would like to thank the Department of Gastroenterology and the Department of Nursing for their support.

\section{References}

[1] Tanaka S, Kashida H, Saito Y, Yahagi N, Yamano H, Saito S, et al. Japan Gastroenterological Endoscopy Society guidelines for colorectal endoscopic submucosal dissection/endoscopic mucosal resection. Dig Endosc. 2020; 32 (2): 219-239.

[2] Tang X, Tan Y, Liu D. Angiolipoma: a rare esophageal submucosal tumor. Gastrointest Endosc. 2017; 86 (2): 399-400.

[3] Shibata M, Kusafuka K, Ono H. A Rare Submucosal Tumor of the Esophagus. Gastroenterology. 2017; 152 (1): e6-e7.

[4] Zhang SL, Du X, Tang XY, Liu D. Submucosal tunneling endoscopic resection for an unusually sized esophageal submucosal tumor protruding into the mediastinum. Rev Esp Enferm Dig. 2019; 111 (9): 710-711.

[5] Hashimoto R, Okuzono T, Akahira J. An Uncommon Gastric Submucosal Tumor with Mucosal Erosion. Clin Gastroenterol Hepatol. 2018; 16 (5): e57-e58.

[6] Liu Q, Ding L, Qiu X, Meng F. Updated evaluation of endoscopic submucosal dissection versus surgery for early gastric cancer: A systematic review and meta-analysis. Int J Surg. 2020; 73: 28-41.

[7] Du Z, Ding W, Chen T. Suitability and efficacy of submucosal tunneling endoscopic resection for the treatment of giant leiomyoma in the middle and lower esophagus. Dis Esophagus. 2019; 32 (12): 55-59.

[8] Hosokawa K, Yoshida S. [Recent advances in endoscopic mucosal resection for early gastric cancer]. Gan To Kagaku Ryoho. 1998; 25 (4): 476-483.

[9] Nishida T, Goto O, Raut CP, Yahagi N. Diagnostic and treatment strategy for small gastrointestinal stromal tumors. Cancer. 2016; 122 (20): 3110-3118.

[10]Nishida T, Blay JY, Hirota S, Kitagawa Y, Kang YK. The standard diagnosis, treatment, and follow-up of gastrointestinal stromal tumors based on guidelines. Gastric Cancer. 2016; 19 (1): 3-14.

[11]Lu J, Jiao T, Li Y, Zheng M, Lu X. Facilitating retroflexed endoscopic full-thickness resection through loop-mediated or rope-mediated countertraction (with videos). Gastrointest Endosc. 2016; 83 (1): 223-228. 
[12] Shi D, Li R, Chen W, Zhang D, Zhang L, Guo R, et al. Application of novel endoloops to close the defects resulted from endoscopic full-thickness resection with single-channel gastroscope: a multicenter study. Surg Endosc. 2017; 31 (2): 837-842.

[13]Pioche M, Bertrand G, Rivory J. Correction: Endoscopic perforation during EMR or ESD: who should take care of the patient? Endosc Int Open. 2018; 6 (3): E382.
[14]Yamamoto H. Endoscopic submucosal dissection--current success and future directions. Nat Rev Gastroenterol Hepatol. 2012; 9 (9): 519-529.

[15]Gotoda T. Endoscopic resection of early gastric cancer. Gastric Cancer. 2007; 10 (1): 1-11.

[16]Cheng XL, Liu H. Gastric adenocarcinoma mimicking a submucosal tumor: A case report. World J Clin Cases. 2019; 7 (19): 3138-3144. 